\title{
INDICES OF HOLOMORPHIC VECTOR FIELDS RELATIVE TO INVARIANT CURVES ON SURFACES
}

\author{
TATSUO SUWA
}

(Communicated by Eric Friedlander)

\begin{abstract}
The local index of a holomorphic vector field relative to a (possibly singular) invariant complex analytic curve on a complex surface is defined and it is shown that, for a compact curve invariant by a one-dimensional singular foliation on a surface, the sum of the indices is equal to its self-intersection number. An interpretation of the indices in terms of holonomy is also given.
\end{abstract}

In [2], C. Camacho and P. Sad defined the index of a holomorphic vector field relative to an invariant non-singular curve and proved an index formula, which was used in showing the existence of a local integral curve for an arbitrary holomorphic vector field with an isolated singularity at the origin in $\mathbb{C}^{2}$. Then A. Lins Neto generalized, in [7], the definition of indices to the case where invariant curves may have singularities and proved an index formula for curves in $\mathbb{P}^{2} \mathbb{C}$, the complex projective plane. The formula was used in the study of the existence problem of algebraic solutions of dimension-one singular foliations on $\mathbb{P}^{2} \mathbb{C}$. As one of the problems, he proposed to generalize his index formula to the case of dimension-one singular foliations on an arbitrary complex surface. The purpose of this note is to remark that in fact his ideas apply to deal with the general case.

In section 1 , we define the local index of the module $E=(v)$ generated by a holomorphic vector field $v$ on $\mathbb{C}^{2}$ relative to an invariant curve $C$. As in [7], we make use of a meromorphic 1-form coming from a decomposition of a holomorphic 1-form $\omega$ which annihilates $v$. In our case, we take a decomposition of $\omega$ with respect to a defining function of $C$ and restrict the meromorphic form to each irreducible component $C_{i}$ of $C$, while in [7], the decomposition is taken from the beginning with respect to a defining function of each $C_{i}$. In either of these two definitions, the index is an analytic invariant and the difference is a topological invariant, which is given in terms of intersection numbers in Proposition (1.4). It is also expressed in terms of some other invariants of curve singularities. An index formula for the indices defined in section 1 is proved in section 2 (Theorem (2.1)). The result is that, if $C$ is a compact curve in a complex surface $X$ invariant by a one-dimensional singular foliation $E$ on $X$, then the sum of the indices of $E$ relative to $C$ is equal

Received by the editors March 30, 1994.

1991 Mathematics Subject Classification. Primary 14B05, 14H20, 32L30, 32S65; Secondary 57R20, 58F18. 
to the self-intersection number $C^{2}$ of $C$. Thus in the presence of a foliation leaving $C$ invariant, $C^{2}$ is localized to the singular points of the foliation. The proof is done by taking an embedded resolution $\pi: \tilde{X} \longrightarrow X$ of $C$, comparing the indices of $E$ relative to $C$ and the indices of the foliation $\tilde{E}$ induced on $\tilde{X}$ from $E$ relative to the proper transform $\tilde{C}$ of $C$ and applying the CamachoSad index formula for $\tilde{E}$ and $\tilde{C}$. Thus the ingredients are essentially in [2] and [7]. Using the adjunction formula, the index formula is restated in Theorem (2.5), which generalizes the index formula in [7]. In section 3, we give an interpretation of the index in terms of holonomy (Proposition (3.1)), which may be known to experts.

Elsewhere [6] a generalization in the higher-dimensional case is studied along the line of D. Lehmann [5] and that will include an alternative proof of the above index formula.

\section{INDICES OF LOGARITHMIC VECTOR FIELDS}

We denote by $\mathscr{O}$ the ring of germs of holomorphic functions in $\mathbb{C}^{2}=$ $\{(x, y)\}$ at the origin 0 . The $\mathscr{O}$-modules of germs of holomorphic vector fields and of holomorphic 1-forms are denoted, respectively, by $\Theta$ and $\Omega$. Let $C$ be the germ of a reduced curve in $\mathbb{C}^{2}=\{(x, y)\}$ at 0 with defining function $f$ in $\mathscr{O}$. We say that a germ $v$ in $\Theta$ is logarithmic for $C$ if $v(f)$ is in $(f)$, the ideal generated by $f$ in $\mathscr{O}\left([1]\right.$, [9]). Let $C=\cup_{i=1}^{r} C_{i}$ be the irreducible decomposition. It is not difficult to see that a germ $v$ in $\Theta$ is logarithmic for $C$ if and only if it is logarithmic for each $C_{i}$ and that this is the case if and only if (a representative of) $v$ is tangential to each $C_{i}$ at each of its non-singular points sufficiently close to 0 . Thus, if $v$ is a logarithmic vector field for $C$, we also say that $C$ is invariant by $v$. Sometimes $C$ is called a separatrix of $v$. For a germ $v$ in $\Theta$, we let $S(v)$ denote (the germ at 0 of $)$ the set $\{(x, y) \mid v(x, y)=0\}$ and call it the singular set of $v$. If we write $v=a \frac{\partial}{\partial x}+b \frac{\partial}{\partial y}$ with $a$ and $b$ in $\mathscr{O}, S(v)$ is the set of common zeros of $a$ and $b$. In what follows we consider germs $v$ with $\operatorname{codim} S(v) \geq 2$, i.e., $S(v) \subset\{0\}$, which is equivalent to saying that $a$ and $b$ are relatively prime in $\mathscr{O}$. A germ $v$ in $\Theta$ defines a dimension-one (non-singular) foliation near 0 outside of $S(v)$. The foliation is equally defined by a 1 -form $\omega$ which annihilates $v$. If $v=a \frac{\partial}{\partial x}+b \frac{\partial}{\partial y}$, we may write $\omega=b d x-a d y$.

(1.1) Lemma. Let $v$ be a germ in $\Theta$ with $\operatorname{codim} S(v) \geq 2$ and $C$ a germ of reduced curve with defining function $f$. Then $v$ is logarithmic for $C$ if and only if there exist germs $g$ and $h$ in $\mathscr{O}$ and $\eta$ in $\Omega$ such that $h$ and $f$ are relatively prime and that

$$
g \omega=h d f+f \eta .
$$

Proof. The lemma is proved in [7] when $f$ is irreducible. We may use the same argument if we note that, if $f$ is reduced, after a suitable change of coordinates $(x, y)$ of $\mathbb{C}^{2}$, we may assume that $\frac{\partial f}{\partial y}$ and $f$ are relatively prime. Thus suppose $v$ is logarithmic for $C$. Then we have $a \frac{\partial f}{\partial x}+b \frac{\partial f}{\partial y}=k f$ for some $k$ in $\mathscr{O}$. We see that $a$ and $f$ are relatively prime, since by assumption, $a$ and $b$ are relatively prime and $\frac{\partial f}{\partial y}$ and $f$ are relatively prime. Hence, by setting $g=\frac{\partial f}{\partial y}, h=-a$ and $\eta=k d x$, we have the identity $\left(^{*}\right)$. Conversely, 
the identity $\left(^{*}\right)$ implies that $h d f \wedge \omega+f \eta \wedge \omega=0$. This shows that $v(f) \in(f)$, since $d f \wedge \omega=-v(f) d x \wedge d y$ and $h$ and $f$ are relatively prime.

Let $v$ be a logarithmic vector field for a reduced curve $C$ with defining function $f$. Also, let $C=\cup_{i=1}^{r} C_{i}$ be the irreducible decomposition of $C$ and $f=f_{1} \cdots f_{r}$ the corresponding irreducible decomposition of $f$ in $\mathscr{O}$. If we denote by $E=(v)$ the submodule of $\Theta$ generated by $v$, we may think of $E$ as (the tangent sheaf of) a one-dimensional singular foliation near 0 in $\mathbb{C}^{2}$. Note that every germ in $E$ is logarithmic for $C$. Thus for each $i, C_{i}-\{0\}$ is a leaf of the foliation. Now we define the index $\operatorname{Ind}_{0}\left(E ; C, C_{i}\right)$ of $E$ relative to the pair $\left(C, C_{i}\right)$ at 0 as follows. First let $\varphi_{i}: \Delta \longrightarrow C_{i}$ be a local uniformization of $C_{i}$, where $\Delta$ is a disk about the origin in $\mathbb{C}$. Consider a decomposition $\left(^{*}\right)$ of $\omega$ with respect to $f$. Since $h$ and $f$ are relatively prime, $\varphi_{i}^{*} \frac{\eta}{h}$ is a meromorphic 1 -form on $\Delta$. We denote by $\operatorname{Res}_{0} \varphi_{i}^{*} \frac{\eta}{h}$ its residue at 0 and we set;

$$
\operatorname{Ind}_{0}\left(E ; C, C_{i}\right)=-\operatorname{Res}_{0} \varphi_{i}^{*} \frac{\eta}{h}=\frac{\sqrt{-1}}{2 \pi} \int_{\gamma} \varphi_{i}^{*} \frac{\eta}{h},
$$

where $\gamma$ is a closed path in $\Delta$ going around 0 once conterclockwise. As in [7], it is shown that the right-hand side in (1.2) depends only on $E, C$ and $C_{i}$. We set

$$
\operatorname{Ind}_{0}(E ; C)=\sum_{i=1}^{r} \operatorname{Ind}_{0}\left(E ; C, C_{i}\right) \text {. }
$$

Thus $\operatorname{Ind}_{0}\left(E ; C_{i}\right)=\operatorname{Ind}_{0}\left(E ; C_{i}, C_{i}\right)$, which is computed using a decomposition of $\omega$ with respect to $f_{i}$.

(1.3) Remarks. $1^{\circ} . \operatorname{Ind}_{0}\left(E ; C_{i}\right)$ is the index defined by Lins Neto in [7]. When $C_{i}$ is non-singular, it also coincides with the index of Camacho and Sad [2].

$2^{\circ}$. We denote by $L$ the link of $C$ at $0 ; L=C \cap S_{\epsilon}^{3}$, where $S_{\epsilon}^{3}$ is a 3-sphere in $\mathbb{C}^{2}$ with center at 0 and small radius $\epsilon$; and by $B \rightarrow L$ the restriction of the closed disk bundle over $C^{\prime}=C-\{0\}$ (closed tublar neighborhood of $C^{\prime}$ in $\mathbb{C}^{2}$ ). Note that $L$ and $B$ are naturally oriented (we give a fiber bundle the local product orientation with fiber coordinates first). We have, by definition and the projection formula,

$$
\operatorname{Ind}_{0}(E ; C)=\frac{\sqrt{-1}}{2 \pi} \int_{L} \frac{\eta}{h}=\left(\frac{1}{2 \pi \sqrt{-1}}\right)^{2} \int_{\Gamma} \frac{d f}{f} \wedge\left(-\frac{\eta}{h}\right),
$$

where $\Gamma$ denotes the boundary of $B$. Later in section 2 , we see that $\operatorname{Ind}_{0}(E ; C)$ represents a "localized self-intersection number" of $C$ at 0 . Noting that $-\frac{\eta}{h}=$ $\frac{d f}{f}$ along the integral curves, the above formula should be compared with the one for the intersection number at 0 of two curves $C$ and $D$ defined by functions $f$ and $g$ without common factors :

$$
(C \cdot D)_{0}=\frac{1}{2 \pi \sqrt{-1}} \int_{L} \frac{d g}{g}=\left(\frac{1}{2 \pi \sqrt{-1}}\right)^{2} \int_{\Gamma} \frac{d f}{f} \wedge \frac{d g}{g},
$$

where $L$ is the link of $C$ and this time $\Gamma$ is also taken as

$$
\Gamma=\{(x, y)|| f(x, y)|=| g(x, y) \mid=\epsilon\}
$$


with the orientation such that $d(\arg f) \wedge d(\arg g)$ is positive ([3], Chapter 5). In [6], the above formula for the index is generalized to the higher-dimensional case.

(1.4) Proposition. In the above situation, we have

$$
\operatorname{Ind}_{0}\left(E ; C, C_{i}\right)=\operatorname{Ind}_{0}\left(E ; C_{i}\right)+\sum_{j \neq i}\left(C_{i} \cdot C_{j}\right)_{0},
$$

where $\left(C_{i} \cdot C_{j}\right)_{0}$ denotes the intersection number of $C_{i}$ and $C_{j}$ at 0.

Proof. Let $g_{j} \omega=h d f_{j}+f_{j} \eta_{j}$ be a decomposition of $\omega$ with respect to $f_{j}$. Note that we may choose $h$ independent of $j$ (see the proof of Lemma (1.1)). If we set $F_{j}=f_{1} \cdots \hat{f}_{j} \cdots f_{r}$ (omit $f_{j}$ ), we have $F_{j} g_{j} \omega=h F_{j} d f_{j}+f \eta_{j}$. Hence we get

$$
g \omega=h d f+f \eta \quad \text { with } \quad g=\sum_{j=1}^{r} F_{j} g_{j} \quad \text { and } \quad \eta=\sum_{j=1}^{r} \eta_{j} .
$$

Therefore, we have

$$
\operatorname{Ind}_{0}\left(E ; C, C_{i}\right)=-\operatorname{Res}_{0} \varphi_{i}^{*} \frac{\eta}{h}=-\operatorname{Res}_{0} \varphi_{i}^{*} \frac{\eta_{i}}{h}-\sum_{j \neq i} \operatorname{Res}_{0} \varphi_{i}^{*} \frac{\eta_{j}}{h} .
$$

Since $\varphi_{i}^{*} \omega=0$, we have

$$
\operatorname{Res}_{0} \varphi_{i}^{*} \frac{\eta_{j}}{h}=-\operatorname{Res}_{0} \varphi_{i}^{*} \frac{d f_{j}}{f_{j}}=-\left(C_{i} \cdot C_{j}\right)_{0} .
$$

(1.5) Example. Let $C$ be a reduced curve with defining function $f$. The vector field $v=\frac{\partial f}{\partial y} \frac{\partial}{\partial x}-\frac{\partial f}{\partial x} \frac{\partial}{\partial y}$ is logarithmic for $C$ and its annihilator is given by $\omega=d f$. We may think of this as a decomposition of $\omega$ with respect to $f$. Thus if we set $E=(v)$, we have $\operatorname{Ind}_{0}\left(E ; C, C_{i}\right)=0$ for each irreducible component $C_{i}$. Hence we get $\operatorname{Ind}_{0}\left(E ; C_{i}\right)=-\sum_{j \neq i}\left(C_{i} \cdot C_{j}\right)_{0}$ (cf. [7], p. 201).

(1.6) Example. Let $E=(v)$ be generated by $v=\lambda x \frac{\partial}{\partial x}+\mu y \frac{\partial}{\partial y}$ with $\lambda$ and $\mu$ non-zero complex numbers, and set $C_{1}=\{y=0\}$ and $C_{2}=\{x=0\}$. The vector field $v$ is logarithmic for $C=C_{1} \cup C_{2}$. Since $\omega=\mu y d x-\lambda x d y$ is a 1-form which annihilates $v$, we have $\operatorname{Ind}_{0}\left(E ; C_{1}\right)=\frac{\mu}{\lambda}$ and $\operatorname{Ind}_{0}\left(E ; C_{2}\right)=\frac{\lambda}{\mu}$. Hence $\operatorname{Ind}_{0}\left(E ; C, C_{1}\right)=\frac{\mu}{\lambda}+1, \operatorname{Ind}_{0}\left(E ; C, C_{2}\right)=\frac{\lambda}{\mu}+1$ and $\operatorname{Ind}_{0}(E ; C)=$ $\frac{(\lambda+\mu)^{2}}{\lambda \mu}$. We may also compute these invariants directly using the decomposition $(y-x) \omega=(\lambda x+\mu y) d(x y)-(\lambda+\mu) x y(d x+d y)$ of $\omega$ with respect to $x y$.

(1.7) Example. Let $E=(v)$ be generated by $v=2 x \frac{\partial}{\partial x}+3 y \frac{\partial}{\partial y}$, and set $C_{i}=\left\{f_{i}=0\right\}$ with $f_{1}=y, f_{2}=x$ and $f_{3}=x^{3}-y^{2}$. We see easily that $v$ is logarithmic for $C=C_{1} \cup C_{2} \cup C_{3}$. We have $\operatorname{Ind}_{0}\left(E ; C_{1}\right)=\frac{3}{2}$ and $\operatorname{Ind}_{0}\left(E ; C_{2}\right)=\frac{2}{3}$. To compute $\operatorname{Ind}_{0}\left(E ; C_{3}\right)$, note that $y \omega=x d f_{3}-3 f_{3} d x$, $\omega=3 y d x-2 x d y$. Since a uniformization for $C_{3}$ is given by $(x, y)=$ $\left(t^{2}, t^{3}\right)$, we have $\operatorname{Ind}_{0}\left(E ; C_{3}\right)=-\operatorname{Res}_{0} \frac{-3 d t^{2}}{t^{2}}=6$. Using $\left(C_{1}, C_{2}\right)_{0}=1$, $\left(C_{2}, C_{3}\right)_{0}=2$ and $\left(C_{3}, C_{1}\right)_{0}=3$, we have $\operatorname{Ind}_{0}\left(E ; C, C_{1}\right)=\frac{3}{2}+3+1=\frac{11}{2}$, $\operatorname{Ind}_{0}\left(E ; C, C_{2}\right)=\frac{2}{3}+1+2=\frac{11}{3}, \operatorname{Ind}_{0}\left(E ; C, C_{3}\right)=6+2+3=11$ and $\operatorname{Ind}_{0}(E ; C)=\frac{11^{2}}{6}$. We may also compute these invariants directly using the 
decomposition $\left(-x^{4}+4 x^{3} y-y^{3}+3 x y^{2}\right) \omega=(2 x+3 y) d f-11 f(d x+d y)$ of $\omega$ with respect to $f=f_{1} f_{2} f_{3}$.

Let $C$ be a germ of reduced curve in $\mathbb{C}^{2}$ at 0 with defining function $f, C=$ $\cup_{i=1}^{r} C_{i}$ the irreducible decomposition of $C$ and $f=f_{1} \cdots f_{r}$ the corresponding irreducible decomposition of $f$. For the pair $\left(C, C_{i}\right)$, we define a non-negative integer $c_{0}\left(C, C_{i}\right)$ as in [4] 1. Thus let $\varphi_{i}: \Delta \longrightarrow C_{i}$ be a uniformization of $C_{i}$ as before. We have $\varphi_{i}^{*} d f=0$. Hence $\varphi_{i}^{*}\left(\frac{d x}{\partial f / \partial y}\right)=-\varphi_{i}^{*}\left(\frac{d y}{\partial f / \partial x}\right)$, and since either $\frac{\partial f}{\partial x}$ or $\frac{\partial f}{\partial y}$ is not divisible by $f_{i}$, it represents a meromorphic 1-form on $\Delta$. We let $c_{0}\left(C, C_{i}\right)$ be the order of the pole at 0 of this meromorphic 1 -form. Note that it is zero if and only if 0 is a non-singular point of $C$. Since $\varphi_{i}^{*} f_{i}=0$, we have $\varphi_{i}^{*} \frac{\partial f}{\partial x}=\varphi_{i}^{*}\left(f_{1} \cdots \frac{\partial f_{i}}{\partial x} \cdots f_{r}\right)$ and $\varphi_{i}^{*} \frac{\partial f}{\partial y}=\varphi_{i}^{*}\left(f_{1} \cdots \frac{\partial f_{i}}{\partial y} \cdots f_{r}\right)$. Hence we see that

$$
c_{0}\left(C, C_{i}\right)=c_{0}\left(C_{i}, C_{i}\right)+\sum_{j \neq i}\left(C_{i} \cdot C_{j}\right)_{0}
$$

We set

$$
c_{0}(C)=\sum_{i=1}^{r} c_{0}\left(C, C_{i}\right) .
$$

This number is related to the Milnor number of $C$ as follows. Suppose $\frac{\partial f}{\partial y}$ and $f$ are relatively prime. Then denoting by $D$ and $C_{y}$, respectively, the divisor of $\frac{\partial f}{\partial y}$ and the $y$-axis, we have

$$
c_{0}\left(C, C_{i}\right)=\left(D \cdot C_{i}\right)_{0}-\left(C_{y} \cdot C_{i}\right)_{0}+1 .
$$

By a similar computation of intersection numbers as in [7], p.218, it is shown that

$$
c_{0}(C)=\mu_{0}(C)+r-1,
$$

where $\mu_{0}(C)$ denotes the Milnor number of $C$ at 0 . Especially we have $c_{0}\left(C_{i}, C_{i}\right)=\mu_{0}\left(C_{i}\right)$, the Milnor number of $C_{i}$ at 0 . We remark that the invariant $c_{0}(C)$ coincides with $2 \delta$ ([4], Appendix I), where $\delta$ is the invariant defined in [10], Chapter IV, $\S 1$, and that the identity (1.9) coincides with the one proved in [8], Theorem 10.5. From Proposition (1.4) and the identities (1.8) and (1.9), we may write

$$
\operatorname{Ind}_{0}\left(E ; C, C_{i}\right)-c_{0}\left(C, C_{i}\right)=\operatorname{Ind}_{0}\left(E ; C_{i}\right)-\mu_{0}\left(C_{i}\right) .
$$

and

$$
\operatorname{Ind}_{0}(E ; C)-\mu_{0}(C)+1=\sum_{i=1}^{r}\left(\operatorname{Ind}_{0}\left(E ; C_{i}\right)-\mu_{0}\left(C_{i}\right)+1\right)
$$

\section{THE INDEX FORMULA}

Let $X$ be a complex analytic surface, i.e., a complex manifold of complex dimension two. The sheaf of germs of holomorphic functions on $X$ is denoted by $\mathscr{O}_{X}$. A dimension-one singular foliation is defined by a rank-one locally free subsheaf $E$ of the tangent sheaf $\Theta_{X}$ of $X$. The singular set $S(E)$ of $E$ is defined to be the set of points in $X$ where the quotient sheaf $\Theta_{X} / E$ 
fails to be $\mathscr{O}_{X}$-free. Thus a dimension-one singular foliation is represented by the following data: (1) an open covering $\left\{U_{\alpha}\right\}_{\alpha \in A}$ of $X,(2)$ (not identically zero) holomorphic vector field $v_{\alpha}$ on each $U_{\alpha}$, and (3) non-vanishing holomorphic function $e_{\alpha \beta}$ on $U_{\alpha} \cap U_{\beta}$ with $v_{\alpha}=e_{\alpha \beta} v_{\beta}$ on $U_{\alpha} \cap U_{\beta}$ for each pair $(\alpha, \beta)$. The sheaf $E$ above is the sheaf of germs of holomorphic sections of the line bundle dual to the one associated to the cocycle $\left\{e_{\alpha \beta}\right\}$. If we set $S\left(v_{\alpha}\right)=\left\{x \in U_{\alpha} \mid v_{\alpha}(x)=0\right\}$, these sets patch together to obtain the analytic set $S(E)$. From now on we assume that $\operatorname{codim} S(E) \geq 2$. Thus $S(E)$ consists of isolated points in $X$. Also, the integral curves of $v_{\alpha}$ in $U_{\alpha}-S\left(v_{\alpha}\right)$ patch together to obtain a decomposition of $X-S(E)$ into a disjoint union of one-dimensional submanifolds (integral curves of $E$ ). Note that a dimensionone singular foliation is also defined as a rank-one locally free subsheaf of the cotangent sheaf $\Omega_{X}$ of $X$ (the annihilator of $E$ ). For more details about singular foliations, we refer to [11]. Let $C$ be a reduced and (globally) irreducible curve in $X$. We say that $C$ is invariant by $E$ if a generator of $E_{p}$ (and hence every germ in $E_{p}$ ) is logarithmic for $C$ at each point $p$ of $C$. Suppose $C$ is invariant by $E$, and set $S(E ; C)=S(E) \cap C$. Note that $S(E ; C)$ contains the set $\operatorname{Sing}(C)$ of singular points of $C$. At each point $p$ in $S(E ; C)$, we have the index $\operatorname{Ind}_{p}(E ; C)$ as defined in section 1 .

(2.1) Theorem. Let $E$ be a dimension-one singular foliation on a complex surface $X$ and $C$ a reduced and irreducible curve in $X$ invariant by $E$. If $C$ is compact, we have

$$
\sum_{p \in S(E ; C)} \operatorname{Ind}_{p}(E ; C)=C^{2} .
$$

First we consider the following situation. Let $C$ be a germ of reduced curve in $\mathbb{C}^{2}$ at 0 with defining function $f$ and $C=\cup_{i=1}^{r} C_{i}$ its irreducible dacomposition. Also, let $v$ be a vector field logarithmic for $C$ and set $E=(v)$. Denoting by $X$ a small neighborhood of 0 in $\mathbb{C}^{2}$, we take an embedded resolution $\pi: \tilde{X} \longrightarrow X$ of the singularity of $C$. Thus $\tilde{X}$ is a complex surface and $\pi$ a proper holomorphic map onto $X$. The divisor of $\pi^{*} f$ can be written as

$$
\sum_{i=1}^{r} \tilde{C}_{i}+\sum_{j=1}^{s} m_{j} D_{j}
$$

where (1) the $\tilde{C}_{i}$ 's are mutually disjoint non-singular curves and for each $i$, $\left.\pi\right|_{\tilde{C}_{i}}: \tilde{C}_{i} \longrightarrow C_{i}$ is a resolution of the singularity of $C_{i},(2)$ each $D_{j}$ is a projective line and $m_{j}$ a positive integer, (3) if we set $D=\cup_{j=1}^{s} D_{j},\left.\pi\right|_{\tilde{X}-D}$ is biholomorphic onto $X-\{0\}$, and (4) each $\tilde{C}_{i}$ intersects with $D$ at one point $q_{i}$ which is a non-singular point of $D$ and the intersection is transverse. The foliation $E$ canonically induces a singular foliation $\tilde{E}$ on $\tilde{X}$ ([2], [7]).

Lemma. In the above situation, we have

$$
\operatorname{Ind}_{0}\left(E ; C, C_{i}\right)=\operatorname{Ind}_{q_{i}}\left(\tilde{E} ; \tilde{C}_{i}\right)+\left(\sum_{j=1}^{s} m_{j} D_{j} \cdot \tilde{C}_{i}\right)_{q_{i}} \text {. }
$$

Proof. A similar assertion for $\operatorname{Ind}_{0}\left(E ; C_{i}\right)$ is proved in [7], p.210. We may prove the lemma along the same line. Thus let

$$
g \omega=h d f+f \eta
$$


be a decomposition for $\omega$ with respect to to $f$. We have

$$
\pi^{*} g \cdot \pi^{*} \omega=\pi^{*} h \cdot d\left(\pi^{*} f\right)+\pi^{*} f \cdot \pi^{*} \eta .
$$

If we denote $\left(\sum_{j=1}^{s} m_{j} D_{j} \cdot \tilde{C}_{i}\right)_{q_{i}}$ by $m$, we may choose local coordinates $(s, t)$ on $\tilde{X}$ about $q_{i}$ so that $\tilde{C}_{i}$ is defined by $s=0$ and $\sum_{j=1}^{s} m_{j} D_{j}$ by $t^{m}=0$ in a neighborhood of $q_{i}$ and that $t$ is a local coordinate on $\tilde{C}_{i}$ about $q_{i}$. Note that the left-hand side of (2.3) is a multiple of a 1 -form defining the foliation $\tilde{E}$ in a neighborhood of $q_{i}$. The right-hand side of $(2.3)$ can be written as

$$
\tilde{h} d s+s \tilde{\eta}
$$

with $\tilde{h}=\pi^{*} h \cdot u t^{m}$ and $\tilde{\eta}=u t^{m} \pi^{*} \eta+\pi^{*} h \cdot d\left(u t^{m}\right)$. Since $h$ and $f$ are relatively prime, $\tilde{h}$ and $s$ are also relatively prime. Hence, denoting the embedding $\tilde{C}_{i} \hookrightarrow \tilde{X}$ by $l$ and setting $\varphi=\pi \circ l$, we have

$$
\begin{aligned}
\operatorname{Ind}_{q_{i}}\left(\tilde{E} ; \tilde{C}_{i}\right) & =-\operatorname{Res}_{0} l^{*} \frac{\tilde{\eta}}{\tilde{h}}=-\operatorname{Res}_{0}\left(\varphi^{*} \frac{\eta}{h}+\frac{d\left(l^{*} u \cdot t^{m}\right)}{l^{*} u \cdot t^{m}}\right) \\
& =\operatorname{Ind}_{0}\left(E ; C, C_{i}\right)-m,
\end{aligned}
$$

since $l^{*} u$ is a unit.

Now, to prove Theorem (2.1), we take a (global) embedded resolution $\pi$ : $\tilde{X} \longrightarrow X$ of the singularities of $C$. Thus $\tilde{X}$ is a complex surface and $\pi$ a proper holomorphic map onto $X$. The total transform of $C$ can be written as

$$
\tilde{C}+\sum_{j=1}^{s} m_{j} D_{j}
$$

where this time (1) $\tilde{C}$ is a compact connected non-singular curve and $\left.\pi\right|_{\tilde{C}}$ : $\tilde{C} \longrightarrow C$ is a resolution of the singularities of $C,(2)$ each $D_{j}$ is a projective line and $m_{j}$ a positive integer, (3) if we set $D=\cup_{j=1}^{s} D_{j},\left.\pi\right|_{\tilde{X}-D}$ is biholomorphic onto $X-\operatorname{Sing}(C)$, and (4) $\tilde{C}$ intersects with $D$ at a finite number of points which are non-singular points of $D$ and each intersection is transverse. The foliation $E$ canonically induces a singular foliation $\tilde{E}$ on $\tilde{X}$. Then by Lemma (2.2), the sum $\sum \operatorname{Ind}(E ; C)$ of the indices of $E$ relative to $C$ is equal to the sum $\sum \operatorname{Ind}(\tilde{E} ; \tilde{C})$ of the indices of $\tilde{E}$ relative to $\tilde{C}$ plus $\sum m_{j} D_{j} \cdot \tilde{C}$, the total intersection number of $\sum m_{j} D_{j}$ and $\tilde{C}$. By the Camacho-Sad index theorem in [2], we have $\sum \operatorname{Ind}(\tilde{E} ; \tilde{C})=\tilde{C}^{2}$. The theorem follows if we apply the projection formula $C^{2}=\tilde{C}^{2}+\sum m_{j} D_{j} \cdot \tilde{C}$.

(2.4) Remark. Thus in the presence of a foliation $E$ leaving $C$ invariant, the self-intersection number is localized to the set $S(E ; C)$, which includes the singular set $\operatorname{Sing}(C)$ of $C$.

Theorem $(2.1)$ is restated as

(2.5) Theorem. Let $X, E$ and $C$ be as in Theorem (2.1). For each $p$ in $S(E ; C)$, let $\cup_{i=1}^{r_{p}} C_{p, i}$ be the irreducible decomposition of $C$ at $p$. Then we have

$$
\sum_{p \in S(E ; C)} \sum_{i=1}^{r_{p}} \operatorname{Ind}_{p}\left(E ; C_{p, i}\right)=-K_{X} \cdot C-\chi(\tilde{C})+\sum_{p \in S(E ; C)} \sum_{i=1}^{r_{p}} \mu_{p}\left(C_{p, i}\right)
$$


and

$$
\sum_{p \in S(E ; C)} \operatorname{Ind}_{p}(E ; C)=-K_{X} \cdot C-\chi(\tilde{C})+\sum_{p \in S(E ; C)} c_{p}(C),
$$

where $K_{X}$ denotes the canonical bundle of $X$ and $\chi(\tilde{C})$ the Euler-Poincaré characteristic of a non-singular model $\tilde{C}$ of $C$.

Proof. These follow from Theorem (2.1), (1.10) and the adjunction formula $([4], 2)$

$$
-\chi(\tilde{C})=C^{2}+K_{X} \cdot C-\sum_{\left.p \in \operatorname{Sing}^{\prime} C\right)} c_{p}(C) .
$$

(2.6) Remarks. $1^{\circ}$. In the right-hand side of each of the above identities, the set $S(E ; C)$ may be replaced by $\operatorname{Sing}(C)$.

$2^{\circ}$. The first formula above is proved in [7], Theorem $\mathrm{A}$, when $X=\mathbb{P}^{2} \mathbb{C}$.

$3^{\circ}$. In Theorems (2.1) and (2.5), $X$ need not be compact.

\section{A REMARK ON THE INDICES}

As is known the Camacho-Sad index can be interpreted in terms of holonomy. In this section, we remark that a similar interpretation is possible for the singular invariant curve case as well.

For simplicity, we consider only (locally) irreducible curves. Thus let $C$ be the germ of a reduced and irreducible curve in $\mathbb{C}^{2}=\{(x, y)\}$ at 0 with defining function $f$. By changing the coordinate system if necessary, we may assume that $f$ is a Weierstrass polynomial in $y$. Thus $C$ has a uniformization $\varphi$ : $\Delta \rightarrow C$ of the form $\varphi(t)=\left(t^{m}, y(t)\right)$ and $f$ and $\frac{\partial f}{\partial y}$ are relatively prime. On $C^{\prime}=C-\{0\}, \frac{\partial f}{\partial y}$ does not vanish and in a neighborhood of $C^{\prime},(x, f)$ form a coordinate system and $x$ is a coordinate on $C^{\prime}$. Let $v$ be a logarithmic vector field for $C$ and $E$ the foliation generated by $v$. In the $(x, f)$ coordinate system, take a point $\left(x_{0}, 0\right)$ on $C$ for sufficiently small $\left|x_{0}\right|$ and consider the complex line $\mathbb{C}_{x_{0}}$ given by $x=x_{0}$. Also for small $\left|f_{0}\right|$, let $P=\left(x_{0}, f_{0}\right)$ be a point on $\mathbb{C}_{x_{0}}$ and let the integral curve through $P$ be $C_{P}$, which is a non-singular (complex) curve in $\mathbb{C}^{2}-\{0\}$. Let $t_{0}$ be a point in $\Delta$ such that $x_{0}=t_{0}^{m}$. A closed path in $\Delta$ based at $t_{0}$ and going around the origin once counterclockwise corresponds, by $\varphi$, to a closed path $\gamma_{0}$ in $C$ based at $\left(x_{0}, 0\right)$ and going around 0 once along the link $L$ of $C$. We denote by $\gamma$ the lift of $\gamma_{0}$ in $C_{P}$ with the initial ponit at $P$. Its end point is in $\mathbb{C}_{x_{0}}$ and can be written as $\left(x_{0}, H\left(f_{0}\right)\right)$, where $H\left(f_{0}\right)$ is a holomorphic function of $f_{0}$ (the holonomy of the foliation at $P$ along $\gamma)$. Since $H(0) \neq 0$, we may write $H\left(f_{0}\right)=\sum_{i \geq 1} c_{i}\left(f_{0}\right)^{i}$. Note that $c_{1}$ is non-zero and its argument is determined by the path $\gamma$.

(3.1) Proposition. In the above situation, we have

$$
\operatorname{Ind}_{0}(E ; C)=\frac{1}{2 \pi \sqrt{-1}} \log c_{1} .
$$

Proof. Consider the decomposition $g \omega=h d f+f \eta$ for an annihilator $\omega$ of $v$ as in Lemma (1.1). Since $g$ and $f$ are also relatively prime, $g$ is nonvanishing in a neighborhood of $C^{\prime}$. Thus the integral curves of $v$ near $C^{\prime}$ are 
obtained by solving the differential equation $h d f+f \eta=0$ or

$$
-\frac{\eta}{h}=\frac{d f}{f} \text {. }
$$

Suppose the integral curve $C_{P}$ is given, in a neighborhood of $P$, by

$$
f=\psi\left(x, f_{0}\right) \text {. }
$$

Thus it satisfies (3.2) and we have $\psi\left(x_{0}, f_{0}\right)=f_{0}$. Now outside of 0 , we may write $-\frac{\eta}{h}=a(x, f) d x+b(x, f) d f$ with $a(x, f)$ and $b(x, f)$ holomorphic in $(x, f)$. From (3.2), we have

$$
\left(a\left(x, \psi\left(x, f_{0}\right)\right)+b\left(x, \psi\left(x, f_{0}\right)\right) \frac{d \psi}{d x}\left(x, f_{0}\right)\right) d x=d \log \psi\left(x, f_{0}\right),
$$

for $x$ near $x_{0}$. If we denote by $A_{0}(x)$ a primitive function of the left-hand side above, fixing the argument of $\psi\left(x, f_{0}\right)$ suitably, we have $A_{0}(x)=\log \psi\left(x, f_{0}\right)$. We consider the analytic continuation of $A_{0}(x)$ along $\gamma$ to get a holomorphic function $A_{1}(x)$ in a neighborhood of $x_{0}$. The analytic continuation of $\psi\left(x, f_{0}\right)$ along $\gamma$ is $\psi\left(x, H\left(f_{0}\right)\right)$ in a neighborhood of $x_{0}$ and its argument is determined from that of $\psi\left(x, f_{0}\right)$. We have

$$
A_{1}(x)-A_{0}(x)=\log \frac{\psi\left(x, H\left(f_{0}\right)\right)}{\psi\left(x, f_{0}\right)} \text {. }
$$

If we set $x=x_{0}$, the left-hand side becomes $-\int_{\gamma} \frac{\eta}{h}$ and the right-hand side $\log \frac{H\left(f_{0}\right)}{f_{0}}$. Finally if we make $f_{0}$ approach 0 , we get $-\int_{\gamma_{0}} \frac{\eta}{h}=\log c_{1}$. Thus we obtain the formula.

\section{REFERENCES}

1. J.W. Bruce and R.M. Roberts, Critical points of functions on analytic varieties, Topology 27 (1988), 57-90.

2. C. Camacho and P. Sad, Invariant varieties through singularities of holomorphic vector fields, Ann. of Math. (2) 115 (1982), 579-595.

3. Ph. Griffiths and J. Harris, Principles of algebraic geometry, Wiley, New York, Chichester, Brisbane, and Toronto, 1978.

4. K. Kodaira, On compact complex analytic surfaces, I, Ann. of Math. (2) 71 (1960), 111-152.

5. D. Lehmann, Résidus des sous-variétés invariantes d'un feuilletage singulier, Ann. Inst. Fourier (Grenoble) 41 (1991), 211-258.

6. D. Lehmann and T. Suwa, Residues of holomorphic vector fields relative to singular invariant subvarieties, preprint.

7. A. Lins Neto, Algebraic solutions of polynomial differential equatuins and foliations in dimension two, Holomorphic Dynamics (Mexico 1986), Lecture Notes in Math., vol. 1345, Springer-Verlag, New York, Heidelberg, and Berlin, 1988, pp. 192-232.

8. J. Milnor, Singular points of complex hypersurfaces, Ann. of Math. Stud., no. 61, Princeton Univ. Press, Princeton, NJ, 1968.

9. K. Saito, Theory of logarithmic differential forms and logarithmic vector fields, J. Fac. Sci. Univ. Tokyo Sect.1 A Math. 27 (1980), 265-291.

10. J.-P. Serre, Groupes algébriques et corps de classes, Hermann, Paris, 1959.

11. T. Suwa, Unfoldings of complex analytic foliations with singularities, Japan. J. Math. (N.S.) 9 (1983), 181-206.

Department of Mathematics, Hokkaido University, Sapporo 060, Japan

E-mail address: suwa@math.hokudai.ac.jp 